PROCEEDINGS OF THE

AMERICAN MATHEMATICAL SOCIETY

Volume 135, Number 6, June 2007, Pages 1631-1640

S 0002-9939(06)08679-5

Article electronically published on December 29, 2006

\title{
ON A CLASS OF COHERENT REGULAR RINGS
}

\author{
CHRISTEL ROTTHAUS AND LIANA M. ŞEGA
}

(Communicated by Bernd Ulrich)

\begin{abstract}
The paper investigates a special class of quasi-local rings. It is shown that these rings are coherent and regular in the sense that every finitely generated submodule of a free module has a finite free resolution.
\end{abstract}

\section{INTRODUCTION}

In a series of papers, W. Heinzer, S. Wiegand and C. Rotthaus investigated a class of quasi-local rings which can be described as a peculiar class of birational extensions of polynomial rings over fields. The construction method used for these rings has been introduced by Akizuki and Nagata in order to produce specific examples of Noetherian local rings. Roughly these rings can be described as follows:

Let $K$ be a field, and let $x, y_{1}, \ldots, y_{n}$ be variables over $K$ and $\tau_{1}, \ldots, \tau_{m} \in x K[[x]]$ power series in $x$ which are algebraically independent over $K(x)$. Fix an element

$$
f \in K\left[x, y_{1}, \ldots, y_{n}, \tau_{1}, \ldots, \tau_{m}\right]
$$

which is algebraically independent over $K\left(x, y_{1}, \ldots, y_{n}\right)$. The polynomial $f$ can be considered as an element of the $(x)$-adic completion $K\left[y_{1}, \ldots, y_{n}\right]_{\left(y_{1}, \ldots, y_{n}\right)}[[x]]$ of the localized polynomial ring $R=K\left[x, y_{1}, \ldots, y_{n}\right]_{\left(x, y_{1}, \ldots, y_{n}\right)}$ and thus can be approximated in a natural way by polynomials $r_{n} \in R$ so that $f-r_{n} \in x^{n} K\left[y_{1}, \ldots, y_{n}\right][[x]]$ for all $n \in \mathbb{N}$. The elements

$$
f_{n}=x^{-n}\left(f-r_{n}\right) \in K\left[y_{1}, \ldots, y_{n}\right][[x]]
$$

are the so-called endpieces of $f$. The rings $B$ under investigation in this paper are obtained by adjoining $f$ and all its endpieces $f_{n}$ to $R$ and by localizing at the maximal ideal which is the contraction of the maximal ideal of $\widehat{R}=K\left[\left[x, y_{1}, \ldots, y_{n}\right]\right]$. In a way, the ring $B$ can be understood as a birational extension of the polynomial ring in $n+2$ variables $x, y_{1}, \ldots, y_{n}, f$ over $K$. Moreover, $B$ can be written as a direct limit of localized polynomial rings in the $n+2$ variables $x, y_{1}, \ldots, y_{n}$ and $f_{n}$ over $K$.

In [3], Heinzer, Wiegand, and Rotthaus showed that a simple flatness criterion on rings essentially of finite type over the field $K$ decides if the constructed ring $B$ is Noetherian or not. In previous papers an emphasis has been put on the construction of Noetherian rings, and in a way these examples are well understood. In the Noetherian case $B$ is a regular local ring of dimension $n+1$ with completion

Received by the editors September 19, 2005 and, in revised form, February 10, 2006.

2000 Mathematics Subject Classification. Primary 13E15, 13C10, 13 D02.

Key words and phrases. Coherent rings, regular rings, modules of finite presentation.

(C)2006 American Mathematical Society 
$\widehat{R}=K\left[\left[x, y_{1}, \ldots, y_{n}\right]\right]$. An appropriate choice of $f$ allows the construction of rings with strange formal fibers. For example, if $f$ is not reduced in the completion $K\left[\left[x, y_{1}, \ldots, y_{n}\right]\right]$, the quotient ring $B /(f)$ is an analytically ramified domain.

Not very much is known in the non-Noetherian case. If $B$ is not Noetherian, the maximal ideal $\mathfrak{m}$ of $B$ is still $n+1$ generated by $x, y_{1}, \ldots, y_{n}$ and the $\mathfrak{m}$-adic completion of $B$ equals the formal power series ring $\widehat{R}=K\left[\left[x, y_{1}, \ldots, y_{n}\right]\right]$. However, if $B$ is not Noetherian then $B$ has Krull dimension $n+2$. The purpose of this paper is to show that $B$ is a coherent regular quasilocal ring in the sense that every finitely generated submodule of a free $B$-module has a finite free resolution. Our proof makes use of the fact that $B$ is the nested union of localized polynomial rings $B_{n}$ in $n+2$ variables over $K$.

The paper is organized as follows. In section 1 we discuss the construction method and the main properties of $B$. In section 2 we prove a main result which shows that every finitely generated submodule of a free $B$-module is of finite presentation. Since $B$ is the nested union of localized polynomial rings of dimension $n+2$, and thus of regular local rings, we show in the last section that every finitely generated submodule of a free $B$-module has projective dimension at most $n+1$.

\section{THE RINGS UNDER CONSIDERATION}

2.1. Construction. Let $K$ be a field, let $x, y_{1}, \ldots, y_{n}$ be variables in degree 1 over $K$ and let $R_{0}=K\left[x, y_{1}, \ldots, y_{n}\right]$ be the polynomial ring in $n+1$ variables over $K$. Set $R=\left(R_{0}\right)_{\left(x, y_{1}, \ldots, y_{n}\right)}$, the localized polynomial ring with maximal ideal $\mathfrak{m}_{R}=\left(x, y_{1}, \ldots, y_{n}\right)$. Let $\tau_{1}, \ldots, \tau_{m} \in x K[[x]]$ be power series over $K$ and assume that the set $\left\{\tau_{1}, \ldots, \tau_{m}\right\}$ is algebraically independent over $K(x)$. Assume also that the $\tau_{i}^{\prime} s$ can be represented as

$$
\tau_{i}=\sum_{j=1}^{\infty} a_{i j} x^{j} \in x K[[x]]
$$

where $a_{i j} \in K$. Let

$$
f\left(x, y_{1}, \ldots, y_{n}, \tau_{1}, \ldots, \tau_{m}\right) \in K\left[x, y_{1}, \ldots, y_{n}, \tau_{1}, \ldots, \tau_{m}\right]
$$

be a polynomial in these variables. It can be considered as a power series in $K\left[y_{1}, \ldots, y_{n}\right][[x]]$. Throughout the paper we assume that $f$ is transcendental over $L=Q(R)$, the fraction field of $R$. Since only the variables $\tau_{1}, \ldots, \tau_{m}$ are of interest for our investigation, we write

$$
f\left(\tau_{1}, \ldots, \tau_{m}\right)=f\left(x, y_{1}, \ldots, y_{n}, \tau_{1}, \ldots, \tau_{m}\right) .
$$

The end pieces $f_{t}$ of $f$ are defined as follows: For all $t \in \mathbb{N}$ set

$$
f_{t}=x^{-t}\left(f-f\left(\sum_{j=1}^{t} a_{1 j} x^{j}, \ldots, \sum_{j=1}^{t} a_{m j} x^{j}\right)\right),
$$

where $f\left(\sum_{j=1}^{t} a_{1 j} x^{j}, \ldots, \sum_{j=1}^{t} a_{m j} x^{j}\right)$ is obtained from $f\left(\tau_{1}, \ldots, \tau_{m}\right)$ by replacing each $\tau_{i}$ by its front piece $\sum_{j=1}^{t} a_{i j} x^{j}$. By the Taylor formula $f_{t}$ is again an element of $K\left[y_{1}, \ldots, y_{n}\right][[x]]$. Moreover, for all $t, k \in \mathbb{N}$ the end pieces $f_{t}$ and $f_{t+k}$ are related as follows:

$$
f_{t}=x^{k} f_{t+k}+r_{t, k}
$$


where $r_{t, k} \in K\left[x, y_{1}, \ldots, y_{n}\right]$. In this paper we have fixed the definition of the remainder $r_{t, k}$. It has been shown in [6, 4.3 Proposition] that for the subsequent construction of $B$ we could have chosen any element $r_{t, k} \in R$ so that $f_{t}-r_{t, k} \in x^{k} \widehat{R}$ and defined the end pieces $f_{t+k}$ accordingly. For all $t \in \mathbb{N}$ set

$$
B_{t}=R\left[f_{t}\right]_{\left(\mathfrak{m}_{R}, f_{t}\right)} .
$$

This ring can be considered as a polynomial ring in $n+2$ variables over $K$, localized at the irrelevant maximal ideal. It is naturally a subring of the power series ring $\widehat{R}=K\left[\left[x, y_{1}, \ldots, y_{n}\right]\right]$. For all $t \in \mathbb{N}$ the inclusion

$$
\iota_{t, 1}: B_{t} \hookrightarrow B_{t+1}
$$

is induced by the identity on $R$ and by $f_{t} \mapsto x f_{t+1}+r_{t, 1}$. The ring of interest is given by the union

$$
B=\bigcup_{t=1}^{\infty} B_{t}=\lim _{t \in \mathbb{N}} B_{t} .
$$

This ring $B$ has been studied in [3. It is relatively easy to decide if $B$ is Noetherian or not. Consider the homomorphism of algebras essentially of finite type over $K$ :

$$
\varphi: R[f] \longrightarrow\left(R\left[\tau_{1}, \ldots, \tau_{n}\right]_{\left(\mathfrak{m}_{R}, \tau_{1}, \ldots, \tau_{m}\right)}\right)_{x}
$$

and let

$$
\psi:\left(R\left[\tau_{1}, \ldots, \tau_{n}\right]_{\left(\mathfrak{m}_{R}, \tau_{1}, \ldots, \tau_{m}\right)}\right)_{x} \longrightarrow \widehat{R}_{x}=K\left[\left[x, y_{1}, \ldots, y_{n}\right]\right]_{x}
$$

denote the naturally embedding. The homomorphism $\psi$ is always faithfully flat 3 . According to [3, Theorem 3.2], $B$ is a Noetherian ring if and only if $\varphi$ is flat at all prime ideals of $\left(R\left[\tau_{1}, \ldots, \tau_{n}\right]_{\left(\mathfrak{m}_{R}, \tau\right)}\right)_{x}$ which lie under prime ideals of $\widehat{R}_{x}$ via $\psi$. Equivalently $B$ is Noetherian if and only if the composition

$$
R[f] \stackrel{\varphi}{\longrightarrow}\left(R\left[\tau_{1}, \ldots, \tau_{n}\right]_{\left(\mathfrak{m}_{R}, \tau\right)}\right)_{x} \stackrel{\psi}{\longrightarrow} \widehat{R}_{x}
$$

is flat. In all cases the maximal ideal $\mathfrak{m}_{B}$ of $B$ is generated by $x, y_{1}, \ldots, y_{n}$, every ideal of $B$ which contains the element $x$ is finitely generated, and the completion of $B$ with respect to the maximal ideal $\mathfrak{m}_{B}$ of $B$ equals $\widehat{R}$. The equation (2.1.1) shows that for all $t, k \in \mathbb{N}$ we have $\left(B_{t}\right)_{x}=\left(B_{t+k}\right)_{x}$. In particular, $B_{x}=\left(B_{t}\right)_{x}$ for all $t \in \mathbb{N}$, and the rings $B$ and $B_{t}$ have the same field of quotients. Another interesting property of the ring $B$ has been studied in 7]. As a consequence of [7, Theorem 2.1] we obtain that $B$ is Noetherian if and only if the Krull dimension of $B$ equals $n+1$. In the non-Noetherian case $B$ has dimension $n+2$.

Our aim is to show that the ring $B$ defined above is a coherent regular quasi-local domain. In the Noetherian case this is a well-known fact about regular local rings. Our proof does not distinguish between the Noetherian and the non-Noetherian case, that is, we will not make use of the nature of the map $\varphi$.

2.2. Example. The following example has been studied extensively in [4] and [5].

As above, let $K$ be a field and consider the localized polynomial ring in 2 variables $R=K[x, y]_{(x, y)}$. Let $\tau \in x K[[x]]$ be an algebraically independent element over $K(x)$ :

$$
\tau=\sum_{i=1}^{\infty} a_{i} x^{i}, \quad a_{i} \in K
$$


For all $t \in \mathbb{N}$ let

$$
\tau_{t}=\sum_{i=t+1}^{\infty} a_{i} x^{i-t}
$$

be the end pieces of $\tau$ in $K[[x, y]]$ and let $q=p_{1} \ldots p_{s} \in(x, y) R$ be a product of finitely many mutually not associated prime elements $p_{i} \in R \backslash(x)$. Set $f=q \cdot \tau$. Then for all $t \in \mathbb{N}$ the end pieces of $f$ are given by

$$
f_{t}=q \cdot \tau_{t}=q\left(\sum_{i=t+1}^{\infty} a_{i} x^{i-t}\right) .
$$

For all $t, k \in \mathbb{N}$, note that there is the following formula relating $f_{t}$ and $f_{t+k}$ :

$$
f_{t}=x^{k} f_{t+k}+q\left(\sum_{j=t+1}^{t+k} a_{j} x^{j-t}\right) .
$$

In this case the remainders are given by: $r_{t, k}=q\left(\sum_{j=t+1}^{t+k} a_{j} x^{j-t}\right)$. Set $B_{t}=$ $R\left[f_{t}\right]_{\left(x, y, f_{t}\right)}$ and $B=\bigcup_{t \in \mathbb{N}} B_{t} \subseteq Q(R)(\tau)$.

Next we show that the map

$$
\varphi: R[f] \longrightarrow\left(R[\tau]_{(x, y, \tau)}\right)_{x}
$$

is not flat. For every prime ideal $P_{i}=\left(p_{i}\right)\left(R[\tau]_{(x, y, \tau)}\right)_{x}$ (which is the contraction of $\left.Q_{i}=p_{i} K[[x, y]]_{x}\right)$ we have that $\varphi^{-1}\left(P_{i}\right)=\left(p_{i}, f\right)$ is a prime ideal of height 2 in $R[f]$. In [4] and [5] it has been shown that $B$ is a 3 -dimensional quasi-local domain with maximal ideal $\mathfrak{m}_{B}=(x, y)$. Moreover, $B$ has exactly $s$ prime ideals of height 2 . These prime ideals are exactly the non-finitely generated primes of $B$.

2.3. Now let $B$ and $B_{t}$ be as defined in section [2.1, with $B \subseteq K\left[\left[x, y_{1}, \ldots, y_{n}\right]\right]$ and with $f\left(x, y_{1}, \ldots, y_{n}, \tau_{1}, \ldots, \tau_{m}\right) \in K\left[x, y_{1}, \ldots, y_{n}, \tau_{1}, \ldots, \tau_{m}\right]$. Recall that the inclusion $\iota_{t, k}: B_{t} \hookrightarrow B_{t+k}$ is the identity on $R$ and maps $f_{t}$ into $x^{k} f_{t+k}+r_{t, k}$. It induces a surjective map

given by

$$
\psi_{t, k}: B_{t}\left[Z_{t, k}\right] \rightarrow B_{t+k}
$$

$$
\left.\psi_{t, k}\right|_{B_{t}}=\iota_{t, k} \quad \text { and } \quad \psi_{t, k}\left(Z_{t, k}\right)=f_{t+k} .
$$

Since $B_{t}$ is a regular local ring of dimension $n+2$, the kernel of $\psi_{t, k}$ is the principal prime ideal generated by the polynomial

$$
h_{t, k}=x^{k} Z_{t+k}+r_{t, k}-f_{t},
$$

and the map $\psi_{t, k}$ induces an isomorphism

$$
\bar{\psi}_{t, k}: \frac{B_{t}\left[Z_{t, k}\right]}{\left(h_{t, k}\right)} \stackrel{\cong}{\longrightarrow} B_{t+k} .
$$

For each triple $(t, k, m) \in \mathbb{N}^{3}$ we have the following commutative diagram:

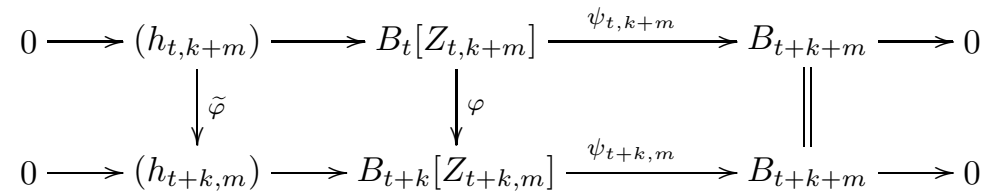

where $\varphi=\varphi_{t, k, m}: B_{t}\left[Z_{t, k+m}\right] \rightarrow B_{t+k}\left[Z_{t+k, m}\right]$ is defined by

$$
\left.\varphi\right|_{B_{t}}=\iota_{t, k} \quad \text { and } \quad \varphi\left(Z_{t, k+m}\right)=Z_{t+k, m}
$$


and the map $\widetilde{\varphi}$ is the restriction of $\varphi$. Note that we can write the ring $B_{t+k+m}$ as a homomorphic image of the ring $B_{t}\left[Z_{t, m+k}\right]$ and as a homomorphic image of $B_{t+k}\left[Z_{t+k, m}\right]$ and that $\varphi$ is an extension of the natural map $\iota_{t, k}: B_{t} \longrightarrow B_{t+k} . \varphi$ maps the kernel of $\psi_{t, k+m}$ into the kernel of $\psi_{t+k, m}$.

It is crucial for the proof in section 3 to understand how $h_{t, k+m}$ relates to $h_{t+k, m}$ under the map $\varphi$.

2.4. Lemma. The following relation holds for all $t, k, m$ :

$$
\widetilde{\varphi}\left(h_{t, m+k}\right)=x^{k} h_{t+k, m} .
$$

Proof. First note that

$$
\begin{aligned}
r_{t, m+k} & =f_{t}-x^{k+m} f_{t+m+k} \\
& =f_{t}-x^{k} f_{t+k}+x^{k} f_{t+k}-x^{k+m} f_{t+m+k} \\
& =r_{t, k}+x^{k} r_{t+k, m} .
\end{aligned}
$$

Thus

$$
\begin{aligned}
\widetilde{\varphi}\left(h_{t, m+k}\right) & =\varphi\left(h_{t, m+k}\right) \\
& =\varphi\left(x^{m+k} Z_{t, m+k}+r_{t, m+k}-f_{t}\right) \\
& =x^{m+k} Z_{t+k, m}+r_{t, m+k}-f_{t} \\
& =x^{m+k} Z_{t+k, m}+r_{t, k}+x^{k} r_{t+k, m}-f_{t} .
\end{aligned}
$$

Using the equation (2.1.1), we further have

$$
\begin{aligned}
\widetilde{\varphi}\left(h_{t, m+k}\right) & =x^{m+k} Z_{t+k, m}+r_{t, k}+x^{k} r_{t+k, m}-f_{t} \\
& =x^{m+k} Z_{t+k, m}+x^{k} r_{t+k, m}-x^{k} f_{t+k} \\
& =x^{k} h_{t+k, m} .
\end{aligned}
$$

\section{Coherence}

A ring $B$ is said to be coherent if every finitely generated ideal of $B$ is of finite presentation. We refer to 2 for basic results concerning this notion.

In this section we show that the $\operatorname{ring} B$ defined in section 2.1 is coherent. Since the proof will also be used in the next section, we show a more general statement, namely that every finitely generated submodule of a finitely generated free $B$ module is of finite presentation.

3.1. Theorem. Let $B \subseteq K\left[\left[x, y_{1}, \ldots, y_{n}\right]\right]$ be a ring as in section 2.1. If $r \in \mathbb{N}$ and $M=\sum_{i=1}^{s} B m_{i} \subseteq B^{r}$ is a finitely generated submodule of $B^{r}$, then $M$ is of finite presentation.

The lemma used in the proof of the theorem is independent of the more complex structure of the ring in section 2.1, hence we prove it in greater generality for a ring $B$ as described below.

3.2. Let $B$ be a commutative ring, and let $\left\{B_{n}\right\}_{n \geq 0}$ be a sequence of Noetherian subrings of $B$ with $B_{n} \subseteq B_{n+1}$ for all $n \in \mathbb{N}$ and such that $B=\bigcup_{n \geq 0} B_{n}$. 
Let $M=\sum_{i=1}^{s} B m_{i} \subseteq B^{r}$ be a finitely generated submodule of the finite free $B$-module $B^{r}$ and let $q \in \mathbb{N}$ be an integer so that

$$
m_{i} \in B_{n}^{r} \quad \text { for all } \quad n \geq q .
$$

For all $n \geq q$ set

$$
M_{n}=\sum_{i=1}^{s} B_{n} m_{i} \subseteq B_{n}^{r} .
$$

Let $\chi: B^{s} \rightarrow M$ denote the $B$-module homomorphism which sends the standard basis of $B^{s}$ to $m_{1}, \ldots, m_{s}$. For each $n \geq q$ let $\chi_{n}: B_{n}^{s} \rightarrow M_{n}$ denote the similarly defined $B_{n}$-homomorphism which is the restriction of $\chi$. Set $L_{n}=\operatorname{Ker}\left(\chi_{n}\right)$ and $L=\operatorname{Ker}(\chi)$. Note that $L=\bigcup_{n \geq q} L_{n}$. For each pair $i, t$ with $i \geq t \geq q$ the following diagram is commutative:

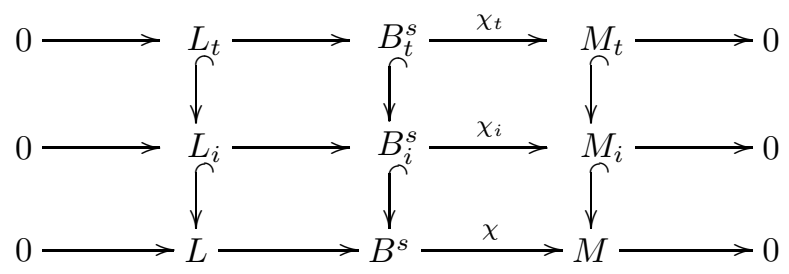

For $i, t$ as above we let

$$
\beta_{t, i}: L_{t} \otimes_{B_{t}} B_{i} \rightarrow L_{i}
$$

denote the map induced by the inclusion $L_{t} \hookrightarrow L_{i}$, and we let

$$
\beta_{t}: L_{t} \otimes_{B_{t}} B \rightarrow L
$$

denote the map induced by the inclusion $L_{t} \hookrightarrow L$.

3.3. Lemma. With the notation in section 3.2, the following hold:

(1) The map $\beta_{t, i}$ is surjective if and only if $\operatorname{Tor}_{1}^{B_{t}}\left(B_{i}, B_{t}^{r} / M_{t}\right)=0$.

(2) If there exists an increasing sequence of integers $\left\{t_{n}\right\}_{n \geq q}$ such that $\beta_{t_{n}, t_{n+1}}$ is surjective for all $n \geq q$, then $\beta_{t}$ is surjective for all $t \geq t_{q}$ and the $B$-module $M$ is of finite presentation.

Proof. (1) For $i, t$ with $i \geq t \geq q$ we have a commutative diagram, with vertical maps induced by the inclusions $M_{t} \hookrightarrow M_{i}, B_{t}^{s} \hookrightarrow B_{i}^{r}$, respectively $L_{t} \hookrightarrow L_{i}$ :

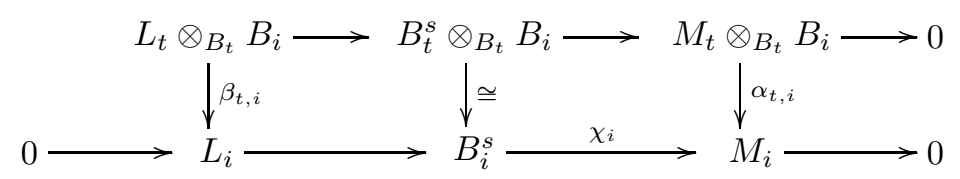

The map $\beta_{t, i}$ is surjective if and only if $\alpha_{t, i}$ is injective. In order to understand the latter map, consider the short exact sequence

$$
0 \rightarrow M_{t} \rightarrow B_{t}^{r} \rightarrow B_{t}^{r} / M_{t} \rightarrow 0
$$

and the induced sequence

$$
0 \rightarrow \operatorname{Tor}_{1}^{B_{t}}\left(B_{t}^{r} / M_{t}, B_{i}\right) \rightarrow M_{t} \otimes_{B_{t}} B_{i} \rightarrow B_{i}^{r} .
$$

Note that the image of the rightmost map is precisely $M_{i}$, and we have a short exact sequence

$$
0 \rightarrow \operatorname{Tor}_{1}^{B_{t}}\left(B_{t}^{r} / M_{t}, B_{i}\right) \rightarrow M_{t} \otimes_{B_{t}} B_{i} \stackrel{\alpha_{t, i}}{\longrightarrow} M_{i} \rightarrow 0 .
$$


In consequence, the map $\beta_{t, i}$ is surjective whenever $\operatorname{Tor}_{1}^{B_{t}}\left(B_{i}, B_{t}^{r} / M_{t}\right)=0$.

(2) Under the given hypothesis, if $\left\{l_{1}, \ldots, l_{d}\right\}$ is a generating set of the $B_{t_{n}}$ module $L_{t_{n}}$ for some $n \geq q$, then $l_{1}, \ldots, l_{d}$ also generate the $B_{t_{k}}$-module $L_{t_{k}}$ for all $k \geq n$. Since $L=\bigcup_{k \geq n} L_{t_{k}}$, it follows that $L$ is generated by $l_{1}, \ldots, l_{d}$. In particular, this shows that $\beta_{t_{n}}$ is surjective for all $n \geq q$. Note that for $t \geq t_{q}$ we have $\beta_{t_{q}}=\beta_{t} \circ\left(\beta_{t_{q}, t} \otimes_{B_{t}} B\right)$. Since $\beta_{t_{q}}$ is surjective, it follows that $\beta_{t}$ is surjective.

In order to prove that a given finitely generated module $M$ is of finite presentation, our approach will be to show that there exists an increasing sequence of integers $\left\{t_{n}\right\}_{n \geq q}$ such that $\operatorname{Tor}_{1}^{B_{t_{n}}}\left(B_{t_{n+1}}, B_{t_{n}}^{r} / M_{t_{n}}\right)=0$ for all $n \geq q$. This will be achieved by using certain maps between homology modules. The purpose of the next paragraph is to define and set up notation for these maps.

3.4. Let $\pi: A \rightarrow A^{\prime}$ be a ring homomorphism, $M, N$ be $A$-modules, $M^{\prime}, N^{\prime}$ be $A^{\prime}$-modules and let $f: M \rightarrow M^{\prime}$ and $g: N \rightarrow N^{\prime}$ be $A$-module homomorphisms. (We make the convention that an $A^{\prime}$-module inherits a structure of an $A$-module via $\pi$.) Let $F$, respectively $G$, be a free $A$-resolution of $M$, respectively $N$, and let $F^{\prime}$, respectively $G^{\prime}$, be a free $A^{\prime}$-resolution of $M^{\prime}$, respectively $N^{\prime}$.

Note that the map $f$ induces a map $M \otimes_{A} A^{\prime} \rightarrow M^{\prime}$, which lifts (uniquely, up to homotopy) to a homomorphism of complexes of $A^{\prime}$-modules $F \otimes_{A} A^{\prime} \rightarrow F^{\prime}$. By composing with the natural homomorphism $F \rightarrow F \otimes_{A} A^{\prime}$, we thus obtain a homomorphism of complexes of $A$-modules $\tilde{f}: F \rightarrow F^{\prime}$. Similarly, we can define $\widetilde{g}: G \rightarrow G^{\prime}$.

$$
\begin{gathered}
\text { With } \operatorname{Tor}^{A}(M, N)=\mathrm{H}\left(F \otimes_{A} G\right) \text { and } \operatorname{Tor}^{A^{\prime}}\left(M^{\prime}, N^{\prime}\right)=\mathrm{H}\left(F^{\prime} \otimes_{A^{\prime}} G^{\prime}\right), \text { we define } \\
\operatorname{Tor}^{\pi}(f, g): \operatorname{Tor}^{A}(M, N) \rightarrow \operatorname{Tor}^{A^{\prime}}\left(M^{\prime}, N^{\prime}\right)
\end{gathered}
$$

to be the map induced in homology by the map $\tilde{f} \otimes_{A} \widetilde{g}: F \otimes_{A} G \rightarrow F^{\prime} \otimes_{A^{\prime}} G^{\prime}$.

When $M=M^{\prime}$ and $f$ is the identity map $M \rightarrow M$, we will write $\operatorname{Tor}^{\pi}(M, g)$ instead of $\operatorname{Tor}^{\pi}(f, g)$.

Proof of Theorem 3.1 . Let $B \subseteq K\left[\left[x, y_{1}, \ldots, y_{n}\right]\right]$ be defined as in section 2.1 and let $M=\sum_{i=1}^{s} B m_{i} \subseteq B^{r}$ be a finitely generated submodule of the finite free module $B^{r}$. We use the notation introduced in section 3.2, with this choice of $B$ and $M$.

Fix an integer $t$ with $t \geq q$ and choose an integer $m=m_{t}$ such that

$$
\left(M_{t}: x^{m}\right)_{B_{t}^{r}}=\left(M_{t}: x^{n}\right)_{B_{t}^{r}} \text { for all } n \geq m .
$$

We will show that $\operatorname{Tor}_{1}^{B_{t+k}}\left(B_{t+k+m}, B_{t+k}^{r} / M_{t+k}\right)=0$ for all $k \geq m$. Then Lemma 3.3 implies that $M$ has a finite presentation.

Let $t, k, m$ be as above and set $p=t+k+m$. Recall that $\iota_{t, k}: B_{t} \rightarrow B_{t+k}$ denotes the natural inclusion, as in Section 1. Since we fixed the indices $t$ and $k$, for the remainder of the proof we will simply denote this map by $\iota$. Consider the following commutative diagram:

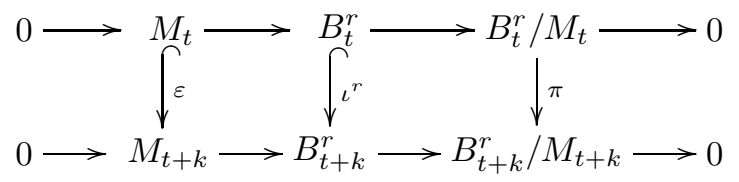

Now apply $\operatorname{Tor}^{B_{t}}\left(B_{p},-\right)$, respectively $\operatorname{Tor}^{B_{t+k}}\left(B_{p},-\right)$, to these short exact sequences and write the long exact sequences in homology. Basic homological algebra 
arguments, as in [1, V 4.3, VI 4.4], show that the following diagram is commutative (the horizontal maps on the left are the connecting homomorphisms):

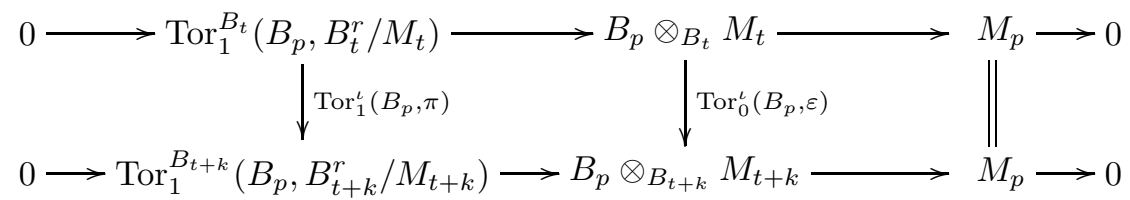

The map $\operatorname{Tor}_{0}^{\iota}\left(B_{p}, \varepsilon\right)$ sends $1 \otimes_{B_{t}} m_{i}$ to $1 \otimes_{B_{t+k}} m_{i}$, for all $i$ with $0 \leq i \leq s$. Recall that for $n \geq q$ the $B_{n}$-module $M_{n}$ is generated by $m_{1}, \ldots, m_{s}$. In consequence, $\operatorname{Tor}_{0}^{\iota}\left(B_{p}, \varepsilon\right)$ is surjective, and the Snake Lemma gives that the map $\operatorname{Tor}_{1}^{\iota}\left(B_{p}, \pi\right)$ is surjective.

In order to prove $\operatorname{Tor}_{1}^{B_{t+k}}\left(B_{p}, B_{t+k}^{r} / M_{t+k}\right)=0$, we will show that the surjective map $\operatorname{Tor}_{1}^{\iota}\left(B_{p}, \pi\right)$ is equal to zero.

To compute the map $\operatorname{Tor}_{1}^{\iota}\left(B_{p}, \pi\right)$, we use the commutative diagram in section 2.3. We apply $\operatorname{Tor}^{B_{t}}\left(-, B_{t}^{r} / M_{t}\right)$, respectively $\operatorname{Tor}^{B_{t+k}}\left(-, B_{t}^{r} / M_{t}\right)$, to the exact sequences in this diagram and we write the induced long exact sequences. As above, we obtain the following commutative diagram:

$$
\begin{gathered}
0 \longrightarrow \operatorname{Tor}_{1}^{B_{t}}\left(B_{p}, \frac{B_{t}^{r}}{M_{t}}\right) \longrightarrow\left(h_{t, k+m}\right) \otimes_{B_{t}} \frac{B_{t}^{r}}{M_{t}} \longrightarrow \frac{B_{t}\left[Z_{t, k+m}\right]^{r}}{M_{t}^{*}} \\
\operatorname{Tor}_{1}^{\iota}\left(B_{p}, \pi\right) \downarrow \\
0 \longrightarrow \operatorname{Tor}_{0}^{\iota}(\widetilde{\varphi}, \pi) \downarrow
\end{gathered}
$$

Here $M_{t}^{*}$ and $M_{t+k}^{*}$ are the submodules of $B_{t}\left[Z_{t, k+m}\right]^{r}$, respectively $B_{t+k}\left[Z_{t+k, m}\right]^{r}$, which are generated by $m_{1}, \ldots, m_{s}$. The vertical map in the middle sends the element $h_{t, k+m} \otimes_{B_{t}} \bar{e}_{j}$ to the element $\widetilde{\varphi}\left(h_{t, m+k}\right) \otimes_{B_{t+k}} \bar{e}_{j}$, where $\bar{e}_{j}$ denotes the image of the canonical basis element $e_{j}$ in $B_{t}^{r} / M_{t}$, respectively $B_{t+k}^{r} / M_{t+k}$.

An element in $\operatorname{Tor}_{1}^{B_{t}}\left(B_{p}, B_{t}^{r} / M_{t}\right)$ has the form

$$
\omega=\sum_{i=0}^{n}\left(b_{i} Z_{t, m+k}^{i} h_{t, k+m} \otimes u_{i}\right), \text { with } b_{i} \in B_{t} \text { and } u_{i} \in B_{t}^{r} / M_{t} .
$$

By Lemma 2.4 we have $\widetilde{\varphi}\left(h_{t, m+k}\right)=x^{k} h_{t+k, m}$ and we obtain

$$
\operatorname{Tor}_{1}^{\iota}\left(B_{p}, \pi\right)(\omega)=\sum_{i=0}^{n}\left(b_{i} Z_{t+m, k}^{i} x^{k} h_{t+k, m} \otimes u_{i}\right) .
$$

We claim that $x^{k} u_{i}=0$ for each $i$. In order to see this, first note that the ideal $\left(h_{t, k+m}\right)$ is a free $B_{t}$-module with basis $\left\{h_{t, k+m} Z^{i}\right\}_{i \geq 0}$. Thus

$$
\left(h_{t, k+m}\right) \otimes_{B_{t}} B_{t}^{r} / M_{t} \cong \bigoplus_{i=0}^{\infty}\left(h_{t, k+m} Z^{i} \otimes B_{t}^{r} / M_{t}\right) \cong \bigoplus_{i=0}^{\infty} B_{t}^{r} / M_{t} .
$$

Since $\left(B_{t+k}\right)_{x}=\left(B_{t}\right)_{x}$, we have

$$
\operatorname{Tor}_{1}^{B_{t}}\left(B_{t+k}, B_{t}^{r} / M_{t}\right)_{x}=0,
$$

and $\omega$ is equal to zero when regarded as an element of $\left(\left(h_{t, k+m}\right) \otimes_{B_{t}} B_{t}^{r} / M_{t}\right)$. 
This implies that for each $i$ we have $u_{i} x^{s_{i}} \in M_{t}$ for some $s_{i} \in \mathbb{N}$; hence

$$
u_{i} \in\left(M_{t}: x^{m}\right)_{B_{t}^{r}} \quad \text { for all } i \text {. }
$$

Since we have chosen $k$ such that $k \geq m$, it follows from 3.1.1 that $x^{k} u_{i}=0$ for all $i$, and hence $\operatorname{Tor}_{1}^{\iota}\left(B_{r}, \pi\right)(\omega)=0$. We thus have $\operatorname{Tor}_{1}^{\iota}\left(B_{r}, \pi\right)=0$, and this finishes the proof, as discussed above.

\section{Regularity}

A coherent ring is said to be regular if every finitely generated submodule of a finitely generated free $B$-module has a finite free resolution. In this section we show that the rings $B$ in section 2.1 are regular.

4.1. Theorem. Let $B \subseteq K\left[\left[x, y_{1}, \ldots, y_{n}\right]\right]$ be a ring as in section 2.1 . If $M$ is a finitely generated submodule of a finitely generated free $B$-module, then $\operatorname{pd}_{B} M \leq$ $n+1$. In particular, $B$ is regular.

Proof. Let $M=\sum_{i=1}^{s} B m_{i} \subseteq B^{r}$ be a finitely generated submodule of the finite free module $B^{r}$. Let $B_{n}=R\left[f_{n}\right]_{\left(\mathfrak{m}_{R}, f_{n}\right)}$ be the localized polynomial rings in $n+2$ variables as in section 2.1. Then $B=\bigcup_{n=1}^{\infty} B_{n}$, and we will further use the notation in section 3.2. We set $L^{1}=L, L_{n}^{1}=L_{n}$ and $s_{0}=s$, where $L, L_{n}$ are as in section 3.2 .

In the proof of Theorem 3.1 we showed that the ring $B$ in section 2.1 satisfies the hypothesis of Lemma 3.3 (2) for a certain strictly increasing sequence $\left\{t_{n}\right\}_{n \geq q}$. In particular, by renaming $B_{t_{n}}$ as $B_{n}$, we may assume that the maps $\beta_{n, n+1}$ : $L_{n} \otimes_{B_{n}} B_{n+1} \rightarrow L_{n+1}$ are surjective for all $n \geq q$, while the equalities $B=\bigcup_{n=q}^{\infty} B_{n}$ and $L^{1}=\bigcup_{n=q}^{\infty} L_{n}^{1}$ still hold. Note that Lemma 3.3 also implies that the map $\beta_{q}: L_{q} \otimes_{B_{q}} B \rightarrow L$ is surjective.

Since $L_{q}^{1}$ is a finitely generated $B_{q}$-module, we have

$$
L_{q}^{1}=\sum_{i=1}^{s_{1}} B_{q} \ell_{1 i} \quad \text { with } \quad \ell_{1 i} \in L_{q}^{1} .
$$

The surjectivity of the maps $\beta_{q, n}$ and $\beta_{q}$ implies that we also have

$$
L_{t}^{1}=\sum_{i=1}^{s_{1}} B_{t} \ell_{1 i} \quad \text { and } \quad L^{1}=\sum_{i=1}^{s_{1}} B \ell_{1 i} .
$$

We will next use the constructions in section 3.2 , only with $L^{1}=\sum_{i=1}^{s_{1}} B \ell_{1 i} \subseteq$ $B^{s_{0}}$ instead of $M=\sum_{i=1}^{s_{0}} B m_{i} \subseteq B^{r}$. For all $t \geq q$ we thus obtain exact sequences

$$
0 \rightarrow L_{t}^{2} \rightarrow B_{t}^{s_{1}} \rightarrow L_{t}^{1} \rightarrow 0
$$

and

$$
0 \rightarrow L^{2} \rightarrow B^{s_{1}} \rightarrow L^{1} \rightarrow 0
$$

with $L^{2}=\bigcup_{n=q}^{\infty} L_{n}^{2}$. As above, we may assume $L^{2}=\sum_{i=1}^{s_{2}} B \ell_{2 i}$ is a finitely generated $B$-module, and $L_{t}^{2}=\sum_{i=1}^{s_{2}} B_{t} \ell_{2 i}$ for each $t \geq q$. Repeating this process we obtain for $t \geq q$ resolutions of $M_{t}$ :

$$
0 \rightarrow L_{t}^{n+1} \rightarrow B_{t}^{s_{n}} \rightarrow B_{t}^{s_{n-1}} \rightarrow \cdots \rightarrow B_{t}^{s_{1}} \rightarrow B_{t}^{s_{0}} \rightarrow M_{t} \rightarrow 0
$$

and for the $B$-module $M$ :

$$
0 \rightarrow L^{n+1} \rightarrow B^{s_{n}} \rightarrow B^{s_{n-1}} \rightarrow \cdots \rightarrow B^{s_{1}} \rightarrow B^{s_{0}} \rightarrow M \rightarrow 0
$$


Again $L^{n+1}=\bigcup_{t \geq q} L_{t}^{n+1}$ and $L^{n+1}=\sum_{i=1}^{s_{n+1}} B \ell_{n+1, i}$ is a finitely generated $B$ module and $L_{t}^{n+1}=\sum_{i=1}^{s_{n+1}} B_{t} \ell_{n+1, i}$ for each $t \geq q$. Each $B_{t}$ is a Noetherian regular local ring of dimension $n+2$. For each $t$, the $B_{t}$-module $M_{t}$ has depth at least 1 (because it is contained in a free module), hence the projective dimension of $M_{t}$ is at most $n+1$. Thus all $n+1$ th syzygies $L_{t}^{n+1}$ are free $B_{t}$-modules. Since for all $t \in \mathbb{N}$ the quotient fields $Q\left(B_{t}\right)$ and $Q(B)$ are identical and since for sufficiently large $t \in \mathbb{N}$ the generators of $L_{t}^{n+1}$ generate the $B$-module $L^{n+1}$, we conclude that $L^{n+1}$ is a free $B$-module. The $B$-module $M$ has thus a finite free resolution:

$$
0 \rightarrow B^{s_{n+1}} \rightarrow B^{s_{n}} \rightarrow \cdots \rightarrow B^{s_{0}} \rightarrow M \rightarrow 0 .
$$

4.2. A projective resolution for the residue field. Let $B \subseteq K\left[\left[x, y_{1}, \ldots, y_{n}\right]\right]$ be again as in section 2.1. Recall that $B$ is a local ring with maximal ideal $\mathfrak{m}_{B}=$ $\left(x, y_{1}, \ldots, y_{n}\right) B$. If $B$ is Noetherian, then a minimal projective resolution of the residue field $K$ is the Koszul complex $\mathcal{K}^{B}$ on the sequence $x, y_{1}, \ldots, y_{n}$. We claim that this remains the case when $B$ is assumed non-Noetherian (and hence the Krull dimension of $B$ is $n+2$ ).

Proposition. The Koszul complex $\mathcal{K}^{B}$ on the sequence $x, y_{1}, \ldots, y_{n}$ is a free resolution of $K$ over $B$. In particular, $\operatorname{pd}_{B} K=n+1$.

Proof. The construction in section 3.2 applied to the module $M:=\mathfrak{m}_{B}=$ $\left(x, y_{1}, \ldots, y_{n}\right) B$ gives that $M_{t}$ is the $B_{t}$-ideal generated by the regular sequence $x, y_{1}, \ldots, y_{n}$. The Koszul complex $\mathcal{K}^{B_{t}}$ on this sequence represents a free resolution of $B_{t} / M_{t}$ over $B_{t}$, and hence $\mathcal{K}_{\geqslant 1}^{B_{t}}$ is a free resolution of $M_{t}$. The argument in the proof of Theorem 4.1 then shows that $\mathcal{K}_{\geqslant 1}^{B}$ is a free resolution of $M\left(=\mathfrak{m}_{B}\right)$ over $B$, hence $\mathcal{K}^{B}$ is a free resolution of $K=B / \mathfrak{m}_{B}$.

\section{REFERENCES}

[1] H. Cartan, S. Eilenberg, Homological Algebra, Princeton Landmarks in Mathematics. Princeton University Press, Princeton, NJ, 1999. MR:1731415 (2000h:18022)

[2] S. Glaz, Commutative coherent rings, Lecture Notes in Mathematics, Vol. 1371, SpringerVerlag, 1989. MR0999133 (90f:13001)

[3] W. Heinzer, C. Rotthaus, S. Wiegand, Noetherian Rings between a semilocal domain and its completion, J. of Alg. 198 (1997), 627-655. MR1489916 (99a:13008)

[4] W. Heinzer, C. Rotthaus, S. Wiegand, Examples of integral domains inside power series rings, Commutative Ring Theory and Applications, Proceedings of the Fourth Conference on Commutative Ring Theory and Applications, Fez, Morocco, Marcel Dekker, 2003. MR2029829 (2004k:13037)

[5] W. Heinzer, C. Rotthaus, S. Wiegand, Non-finitely generated prime ideals in subrings of power series rings, Rings, Modules, Algebras, and Abelian Groups, Proceedings of the Algebra Conference, Venice, Italy, Marcel Dekker, 2004. MR2050721 (2005d:13036)

[6] W. Heinzer, C. Rotthaus, S. Wiegand, Intermediate rings between a local domain and its completion, Illinois J. of Math. 43 (1999), 19-46. MR1665712 (99m:13047)

[7] W. Heinzer, C. Rotthaus, S. Wiegand, Intermediate rings between a local domain and its completion II, Illinois J. of Math. 45 (2001), 965-979. MR.1879247 (2003e:13003)

Department of Mathematics, Michigan State University, East Lansing, Michigan 48824

E-mail address: rotthaus@math.msu.edu

Department of Mathematics and Statistics, University of Missouri, Kansas City, Missouri 64110-2499

E-mail address: segal@umkc.edu 